\title{
Backstepping Control Based on Constrained Command Filter for Hypersonic Flight Vehicles with AOA and Actuator Constraints
}

\author{
Junbao Wei $\mathbb{D},{ }^{1}$ Haiyan Li $\mathbb{D},{ }^{2}$ Ming Guo $\mathbb{D},{ }^{3}$ Jing Li $\mathbb{D}^{2},{ }^{2}$ and Huang Huang $\mathbb{D}^{3}$ \\ ${ }^{1}$ Graduate College, Naval University of Engineering, Wuhan 430000, China \\ ${ }^{2}$ College of Weaponry Engineering, Naval University of Engineering, Wuhan 430000, China \\ ${ }^{3}$ College of Nuclear Science and Technology, Naval University of Engineering, Wuhan 430000, China \\ Correspondence should be addressed to Haiyan Li; haiyanli1818@163.com and Ming Guo; morpheustwo@163.com
}

Received 22 April 2021; Revised 15 August 2021; Accepted 17 August 2021; Published 3 September 2021

Academic Editor: Shaoming He

Copyright (c) 2021 Junbao Wei et al. This is an open access article distributed under the Creative Commons Attribution License, which permits unrestricted use, distribution, and reproduction in any medium, provided the original work is properly cited.

An antisaturation backstepping control scheme based on constrained command filter for hypersonic flight vehicle (HFV) is proposed with the consideration of angle of attack (AOA) constraint and actuator constraints of amplitude and rate. Firstly, the HFV system model is divided into velocity subsystem and height subsystem. Secondly, to handle AOA constraint, a constrained command filter is constructed to limit the amplitude of the AOA command and retain its differentiability. And the constraint range is set in advance via a prescribed performance method to guarantee that the tracking error of the AOA meets the constraint conditions and transient and steady performance. Thirdly, the proposed constrained command filter is combined with the auxiliary system for actuator constraints, which ensures that the control input meets the limited requirements of amplitude and rate, and the system is stable. In addition, the tracking errors of the system are proved to be ultimately uniformly bounded based on the Lyapunov stability theory. Finally, the effectiveness of the proposed method is verified by simulation.

\section{Introduction}

Hypersonic flight vehicle (HFV) has attracted wide attention in the world due to its great application prospect in military and civil fields. As the key technology to develop HFV, flight control technology can realize tracking trajectory and stable flight. Therefore, the research on the controller design of HFV is significant to its development.

HFV is characterized by large flight envelope, complex flight characteristics, and variable external environment and is strong coupled, nonlinear, and uncertain [1]. It is challenging to design a controller for HFV. For the problem of nonminimum phase, Ref. [2] proposes a control-oriented model which ignored the lift-lift coupling. At present, the commonly used idea is to design controllers for the inputoutput subsystem based on output redefinition method [ 3 , 4]. An alternative method which is available for dealing with the controller design problem of such a strong nonlinear system as HFV is backstepping approach [5-11]. The backstepping method is applied firstly to the attitude control of HFV in Ref. [5], and the control design is completed, and the stable tracking of the system is realized. The problem of "differential explosion" occurs easily in the design of the backstepping controller due to the high order of the altitude subsystem of HFV. To solve the problem, the command filter [6], dynamic surface control $[8,9]$, tracking differentiator $[10,11]$, and other technologies are widely used in the backstepping control method. To deal with uncertainties as well as external disturbance of HFV, disturbance observers [12, 13] and intelligent approximations [14-16] are widely used in controller design. In addition, in order to improve the transient performance of the control system, the prescribed performance method is applied in the controller design [17-19]. This method originally proposed by Bechlioulis and Rovithakis [20], which could characterize the convergence rate and maximum overshoot of tracking error such that the desired transient performance is achievable by limiting the tracking error in the prescribed convergence range. Although a lot of progress has been made in the controller design of HFV, the AOA constraint has not gotten 
enough attention. The scramjet engine used in HFV is extremely sensitive to $\mathrm{AOA}$, and its intake efficiency depends on the AOA. The amplitude of AOA must meet certain constraints to ensure the normal operation of the scramjet, otherwise it will cause scramjet thermal choke problem [21]. The traditional method that makes AOA meet the constraint requirements is to make AOA converge to a desired trim value $[22,23]$. However, this method cannot prove that the AOA would be guaranteed to fall into predefined interval in theory. At present, barrier Lyapunov function (BLF) has been effectively used in dealing with the problem of AOA constraint [24-29]. The main characteristic of BLF is that its value tends to infinity as the system approaches a predefined boundary. The boundedness of the BLF indicates that the AOA error is constrained within the specified range. Ref. [24] proposes a modified adaptive dynamic surface control scheme. An asymmetric BLF is introduced in this scheme to guarantee state constraints of HFV. However, in the above literatures on the AOA constraint, the proposed control schemes need to assume that the initial error of AOA satisfies the constraint condition. In practice, the initial error of AOA is difficult to obtain accurately. In addition, most studies focus on the steadystate performance of AOA error and ignore its dynamic performance. If the tracking error has a good dynamic performance, the AOA constraint problem will be better handled and the maneuverability of HFV will be further improved.

In practice, the control force provided by the actuator is limited. It is easy to cause the problem of actuator saturation due to the high-altitude flight of HFV and the influence of the external environment [30]. The ideal control law cannot be effectively implemented when the system is saturated, which leads to large deviation in command tracking and even seriously affecting the stability of the system [31]. Therefore, the input saturation problem cannot be ignored. The commonly method is to construct the auxiliary system in the controller design process [31-35]. The auxiliary variables in the system compensate the tracking error and stabilize the system when actuators are saturated. However, the existence of auxiliary variables affects the convergence of tracking errors inevitably. Therefore, Refs. [33, 34] construct new auxiliary system whose auxiliary variable can converge with faster speed and higher precision when actuator exits saturation. However, the above studies only consider the amplitude saturation of the control input. In practice, the rate of actuators of HFV has also certain limitations. Ref. [36] further considers the saturation of the actuator's amplitude and rate at the same time. The command filter is constructed to constrain the control input, but this method lacks theoretical proof, and the constraint effect of filter is difficult to guarantee in [36]. Ref. [37] proposes an adaptive control scheme with multiple constraints on the amplitude and rate of the actuator. Although the amplitude and rate of the control input are constrained effectively by this scheme, the controller design is too complicated.

In general, in order to solve the control problem of HFV considering AOA limitation and actuator constraint of amplitude and rate, an antisaturation backstepping control strategy based on the constrained command filter is pro- posed. Compared with the existing research work, the main contributions of this paper are as follows:

(1) A control method combining the constrained command filter and prescribed performance method is proposed to handle the problem of AOA constraint. Compared with Refs. [24-29], there is no need to assume that the initial error of AOA is within the limited range in this method, which is easier to implement in practice. In addition, the transient performance of AOA error is further improved, and the theoretical proof that AOA meets the constraint conditions is completed

(2) A control method combining constrained command filter and auxiliary system is proposed to handle actuator constraint. Different from Refs. [31-35], this paper further considers the saturation of the actuator's rate. The control input can satisfy the limit conditions of amplitude and rate in this paper. Compared with Ref. [36, 37], the control law design in this paper is simpler and easier to implement in practice, and the theoretical proof is completed to guarantee the effectiveness of proposed method

The rest of this paper is organized as follows: Section 2 formulates the HFV model and preliminaries; Section 3 presents the controller design process; Sections 4 and 5 give a stability analysis and a simulation study, respectively; the conclusions are proposed in Section 6.

\section{HFV Model and Preliminaries}

2.1. HFV Model. In this paper, we consider the longitudinal control-oriented FHV model developed by Ref. [2]:

$$
\begin{gathered}
\dot{V}=\frac{T \cos \alpha-D}{m}-g \sin \gamma, \\
\dot{h}=V \sin \gamma, \\
\dot{\gamma}=\frac{L+T \sin \alpha}{m V}-\frac{g \cos \gamma}{V}, \\
\dot{\alpha}=q-\dot{\gamma}, \\
\dot{q}=\frac{M}{I_{y y}},
\end{gathered}
$$

where velocity $V$, altitude $h$, flight-path $\gamma, \operatorname{AOA} \alpha$, and pitch rate $q$ are rigid body states; $m, g$, and $I_{y y}$ represent vehicle mass, acceleration owing to gravity, and moment of inertia, respectively; and $T, D, L$, and $M$ represent thrust, drag force, lift, and pitch moment, respectively, with the following expressions [18]: 


$$
\left\{\begin{array}{l}
D \approx Q S\left(C_{D}^{\alpha^{2}} \alpha^{2}+C_{D}^{\alpha} \alpha+C_{D}^{0}\right) \\
L=L_{0}+L_{\alpha} \alpha \\
T=T_{\Phi}(\alpha) \Phi+T_{0}(\alpha) \\
M=M_{T}+M_{0}(\alpha)+M_{\delta_{e}} \delta_{e}
\end{array}\right.
$$

where $Q=(1 / 2) \rho V^{2}$ represents dynamic pressure, in which $\rho$ is air density; $S$ represents reference area; $\Phi, \delta_{e}$ represent deflection fuel equivalency ratio and elevator angular deflection, respectively; $C_{D}^{\alpha^{2}}, C_{D}^{\alpha}, C_{D}^{0}, L_{0}, L_{\alpha}, T_{\Phi}(\alpha), T_{0}(\alpha)$ represent the related aerodynamic parameters of thrust, drag force, and lift, respectively; and $M_{T}, M_{0}\left(\alpha\right.$.), and $M_{\delta_{e}}$ represent the related parameters of pitch moment.

Assumption 1. The value of the term $T \sin \alpha$ in equation (3) is much smaller than the value of lift $L$, so this term can be ignored.

The assumption can be verified by calculation according to the model data and the range of states provided in Ref. [2]. In addition, the rationality of the assumption is also explained and analyzed in Ref. [38].

The outputs of the system model are velocity $V$ and altitude $h$; the control inputs are fuel equivalent ratio $\Phi$ and elevator angular deflection $\delta_{e}$. According to the models (1)-(5) and Assumption 1, it can be seen that the velocity $V$ change is mainly controlled by the fuel equivalent ratio $\Phi$, and the elevator angular deflection $\delta_{e}$ controls the altitude $h$ by directly controlling the change of the pitch rate $q$ and then controlling the change of AOA $\alpha$ and flight-path $\gamma$. In order to facilitate the design of the control law, the models (1)-(5) can be decomposed into velocity subsystem and height subsystem under normal circumstances [39]:

$$
\begin{gathered}
\dot{V}=f_{V}+g_{V} \Phi+d_{1}, \\
\left\{\begin{array}{l}
\dot{h}=V \sin \gamma \\
\dot{\gamma}=f_{\gamma}+g_{\gamma} \alpha+d_{2}, \\
\dot{\alpha}=f_{\alpha}+q-d_{2} \\
\dot{q}=f_{q}+g_{q} \delta_{e}+d_{3},
\end{array}\right.
\end{gathered}
$$

where

$$
\left\{\begin{array}{l}
f_{V}=\frac{T_{0}(\alpha) \cos \alpha-D}{m}-g \sin \gamma, g_{V}=\frac{T_{\Phi}(\alpha) \cos \alpha}{m} \\
f_{\gamma}=\frac{L_{0}-m g \cos \gamma}{m V}, g_{\gamma}=\frac{L_{\alpha}}{m V} \\
f_{\alpha}=-f_{\gamma}-g_{\gamma} \alpha \\
f_{q}=\frac{M_{T}+M_{0}(\alpha)}{I_{y y}}, g_{q}=\frac{M_{\delta_{e}}}{I_{y y}}
\end{array}\right.
$$

$d_{i}(i=1,2,3)$ represent disturbance items, including external interference and parameter perturbation, and the following assumption is made:

Assumption 2. The first derivatives of disturbance terms $d_{i}($ $i=1,2,3$ ) are bounded.

Assumption 2 is common in existing results for the longitudinal motion model of HFV, and the analysis of literatures has shown that this assumption is in line with the actual physical system and external flight environment of HFV, such as Refs. [24, 33].

2.2. Actuator Saturation and AOA Constraint. In order to avoid the phenomenon of thermal choke in the actual project, the fuel equivalent ratio $\Phi$ needs to be within a certain range, otherwise scramjet will stop working [34]. The restricted fuel equivalent ratio $\Phi$ can be described as

$$
\Phi=H_{\Phi}\left(\Phi_{d}\right)=\left\{\begin{array}{l}
\Phi_{\max }, \Phi_{d} \geq \Phi_{\max } \\
\Phi_{d}, \Phi_{\min }<\Phi_{d}<\Phi_{\max } \\
\Phi_{\min }, \Phi_{d} \leq \Phi_{\min }
\end{array}\right.
$$

where $\Phi_{d}$ represents the ideal control law and $\Phi_{\max }$ and $\Phi_{\text {min }}$ are the upper and lower bounds of $\Phi$, respectively.

The amplitude and rate of the elevator angular deflection $\delta_{e}$ are limited due to the deflection limit of actual physical mechanism, and the limited conditions can be described as

$$
\begin{gathered}
\delta_{e}=H_{\delta_{e}}\left(\delta_{e d}\right)=\left\{\begin{array}{l}
\delta_{\max }, \delta_{e d} \geq \delta_{\max }, \\
\delta_{e d}, \delta_{\min }<\delta_{e d}<\delta_{\max }, \\
\delta_{\min }, \delta_{e d} \leq \delta_{\min },
\end{array}\right. \\
\Psi_{e}=H_{\Psi}\left(\Psi_{e d}\right)=\left\{\begin{array}{l}
\Psi_{\max }, \Psi_{e d} \geq \Psi_{\max }, \\
\Psi_{e d}, \Psi_{\min }<\Psi_{e d}<\Psi_{\max }, \\
\Psi_{\text {min }}, \Psi_{e d} \leq \Psi_{\min },
\end{array}\right.
\end{gathered}
$$

where $\delta_{e d}$ represents the ideal control law; $\delta_{\max }$ and $\delta_{\min }$ are the upper and lower bounds of $\delta_{e}$, respectively; $\Psi_{e}=\dot{\delta}_{e}$ is the rate of elevator angular deflection; $\Psi_{e d}=\dot{\delta}_{e d}$ is the rate of the ideal control law; and $\Psi_{\max }$ and $\Psi_{\min }$ are the upper and lower bounds of $\Psi_{e}$, respectively.

The scramjet of HFV is extremely sensitive to the AOA which directly affects its operating conditions [21]. The normal operation of the scramjet requires $\mathrm{AOA}$ to be in an allowable range. So it is necessary to consider AOA constraint which can be expressed as

$$
A_{m} \leq \alpha \leq A_{M}
$$

where $A_{M}$ and $A_{m}$ are the upper and lower bounds of AOA, respectively.

2.3. Prescribed Performance. The prescribed performance [20] method includes performance function and error transformation, which means that while the tracking error 
converges in an arbitrary small set, the convergence rate and overshoot of the tracking error meet the prescribed conditions.

Performance function $\omega(t)$ is a strictly decreasing positive function. Then, the objective of guaranteeing prescribed tracking performance is equivalent to ensure that

$$
-\omega(t)<e(t)<\omega(t)
$$

To transform inequality constraints into equality constraints, define

$$
e(t)=\omega(t) S(\varepsilon)
$$

where $\varepsilon$ denotes transformed error. The conversion function is defined as $S(\varepsilon)=\left(e^{\varepsilon}-e^{-\varepsilon}\right) /\left(e^{\varepsilon}+e^{-\varepsilon}\right)$. It is easy to know that $S(\varepsilon)$ is a smooth and strictly increasing reversible function with the following properties:

$$
\left\{\begin{array}{l}
-1<S(\varepsilon)<1, \\
\lim _{\varepsilon \longrightarrow-\infty} S(\varepsilon)=-1, \\
\lim _{\varepsilon \longrightarrow \infty} S(\varepsilon)=1 .
\end{array}\right.
$$

Further, the inverse transformation of $S(\varepsilon)$ is defined as

$$
\varepsilon=S^{-1}(e, \omega)=\frac{1}{2} \ln \left(\frac{1+e / \omega}{1-e / \omega}\right) .
$$

Remark 3. According to equation (17), if $\varepsilon$ is bounded, then inequality (14) holds. That is, the tracking error of system is not only bounded but also limited within the setting range, which guarantees that it meets the requirements of prescribed transient and steady-state performance.

2.4. Command Filter. The command filter in [40] is as follows:

$$
\left\{\begin{array}{l}
\dot{\chi}_{1}=\chi_{2}, \\
\dot{\chi}_{2}=2 \tau \omega\left[\frac{\omega^{2}}{2 \tau \omega}\left(x_{d}-\chi_{1}\right)-\chi_{2}\right]
\end{array}\right.
$$

where $x_{d}$ is the filter input; the outputs $\chi_{1}$ and $\chi_{2}$ are the estimated value of $x_{d}$ and its first derivative; the filter design parameters are $\omega>0$ and $\tau \in(0,1]$.

According to Ref. [40], there are $\lim _{\omega \longrightarrow \infty} \chi_{1}=x_{d}$ and $\lim _{\omega \longrightarrow \infty} \chi_{2}=\dot{x}_{d}$. Therefore, the following assumption is made.

Assumption 4. There exist unknown constants $\eta_{1}>0, \eta_{2}>0$ such that $\left|x_{1}-x_{d}\right| \leq \eta_{1}$ and $\left|x_{2}-\dot{x}_{d}\right| \leq \eta_{2}$.

2.5. Linear Extended State Observer. Consider the following first-order uncertain system:

$$
\left\{\begin{array}{l}
\dot{x}_{1}(t)=f\left(x_{1}\right)+g\left(x_{1}\right) u+d(t) \\
y=x_{1}(t)
\end{array}\right.
$$

where $d(t)$ represents the uncertain term, it is assumed that $d(t)$ is continuous, and its first derivative is bounded. For this system, LESO can be established as shown below:

$$
\left\{\begin{array}{l}
e_{1}(t)=x_{1}(t)-\hat{x}_{1}(t), \\
\dot{\hat{x}}_{1}(t)=\hat{x}_{2}(t)+l_{1} e_{1}(t)+f\left(x_{1}\right)+g\left(x_{1}\right) u \\
\dot{\hat{x}}_{2}(t)=l_{2} e_{1}(t),
\end{array}\right.
$$

where $\widehat{x}_{1}(t)$ is the estimation value of $x_{1}(t) ; \widehat{x}_{2}(t)$ is the estimation value of $d(t)$; and $l_{i}>0(i=1,2)$.

The bandwidth configuration method [41] is used to select the parameters which meet the following conditions: $\left[l_{1}, l_{2}\right]=\left[\omega_{0} \alpha_{1}, \omega_{0} \alpha_{2}\right]$, where $\omega_{0}$ is the bandwidth of the observer, and the parameters are chosen as $\alpha_{i}=3 ! / i ! \bullet(3-i)$ $!(i=1,2)$. According to Ref. [42] on the proof of LESO convergence that the observation error $\tilde{d}=\widehat{x}_{2}-d$ converges to zero in a finite time, the assumption can be made as follows.

Assumption 5. LESO estimation error is bounded, and there exists an unknown constant $\tilde{D}>0$, such that $|\tilde{d}| \leq \tilde{D}$.

\section{Controller Design}

For the control objective, considering that the control system of HFV with AOA constraint and actuator constraints of amplitude and rate, the system output can track the command signal stably, and the actuators and AOA meet the constraints. The structure of control scheme presented for FHV is shown in Figure 1.

3.1. Velocity Subsystem Controller Design. According to equation (7), the tracking error is defined as $e=V-V_{d}$, where $V_{d}$ is the velocity command. The compensation error is defined as

$$
v_{V}=e_{V}-\xi_{V}
$$

where $\xi_{V}$ is the auxiliary variable to be designed.

Combining equation (7) and deriving equation (21), we get

$$
\dot{v}_{V}=f_{V}+g_{V} \Phi+d_{1}-\dot{V}_{d}-\dot{\xi}_{V}
$$

The subsystem control law is designed as

$$
\Phi_{d}=\frac{1}{g_{V}}\left(-k_{V} v_{V}-f_{V}+\dot{V}_{d}-\widehat{d}_{1}-k_{V \xi} \xi_{V}\right),
$$

where $k_{V}>0, k_{V \xi}>0$, and $\widehat{d}_{1}$ is the LESO estimation of $d_{1}$.

Considering the input saturation problem of fuel equivalent ratio $\Phi$, by substituting equation (23) into equation (10), the actual control input can be obtained:

$$
\Phi=H_{\Phi}\left(\Phi_{d}\right)
$$




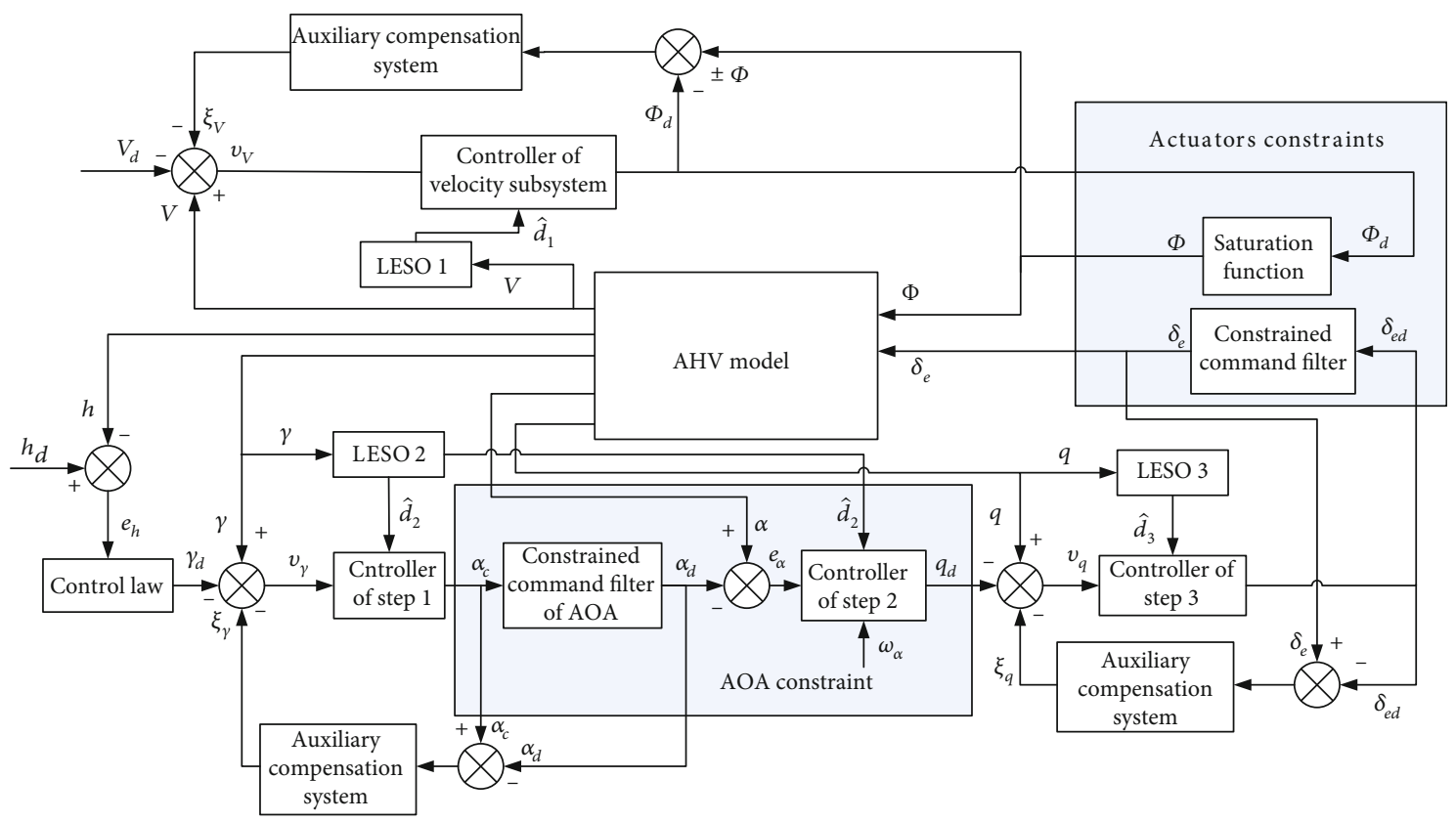

Figure 1: The structure of control scheme presented for FHV.

In order to guarantee the stability of system when fuel equivalent ratio $\Phi$ is saturated, the auxiliary compensation system is designed as

$$
\dot{\xi}_{V}=-k_{V \xi} \xi_{V}+g_{V}\left(\Phi-\Phi_{d}\right)
$$

Substituting equations (23)-(25) into equation (22), we get

$$
\dot{v}_{V}=-k_{V} v_{V}+d_{1}-\widehat{d}_{1}
$$

3.2. Height Subsystem Controller Design. Define the height tracking error $e_{h}=h-h_{d}$, where $h_{d}$ is the height command. According to Ref. [24], the virtual control law is designed as

$$
\gamma_{d}=\arcsin \left(\frac{-k_{h} e_{h}+\dot{h}_{d}}{V}\right) \text {, }
$$

where $k_{h}>0$ is the designed parameter.

Define the errors of flight path, AOA, and pitch rate, respectively

$$
e_{\gamma}=\gamma-\gamma_{d}, e_{\alpha}=\alpha-\alpha_{d}, e_{q}=q-q_{d}
$$

where $\alpha_{d}$ and $q_{d}$ are the virtual control laws to be designed.

Considering that it is difficult to obtain the derivative of the virtual law in the design of the backstepping controller, the command filter is introduced to estimate the virtual command $\gamma_{d}$ and its derivative $\dot{\gamma}_{d}$.

$$
\left\{\begin{array}{l}
\dot{\chi}_{\gamma 1}=\chi_{\gamma 2}, \\
\dot{\chi}_{\gamma 2}=2 \tau_{\gamma} \omega_{\gamma}\left[\frac{\omega_{\gamma}^{2}}{2 \tau_{\gamma} \omega_{\gamma}}\left(\gamma_{d}-\chi_{\gamma 1}\right)-\chi_{\gamma 2}\right]
\end{array}\right.
$$

where $\tau_{\gamma}>0$ and $\omega_{\gamma}>0$.

Step 1. Define compensation error

$$
v_{\gamma}=e_{\gamma}-\xi_{\gamma}
$$

where $\xi_{\gamma}$ is the auxiliary variable to be designed.

Combining equation (8) and deriving (30), we get

$$
\dot{v}_{\gamma}=f_{\gamma}+g_{\gamma} \alpha_{d}+g_{\gamma} e_{\alpha}+d_{2}-\dot{\gamma}_{d}-\dot{\xi}_{\gamma} \text {. }
$$

Considering the problem of AOA constraint, the desired control law $\alpha_{c}$ needs to be limited; and in order to ensure the differentiability of the limiting command, the following constrained command filter is constructed to obtain the control law $\alpha_{d}$ :

$$
\left\{\begin{array}{l}
\dot{\chi}_{\alpha 1}=\chi_{\alpha 2} \\
\dot{\chi}_{\alpha 2}=2 \tau_{\alpha} \omega_{\alpha}\left[\frac{\omega_{\alpha}^{2}}{2 \tau_{\alpha} \omega_{\alpha}}\left(\operatorname{sat}\left(\alpha_{c}\right)-\chi_{\alpha 1}\right)-\chi_{\alpha 2}\right] \\
\alpha_{d}=\chi_{\alpha 1},
\end{array}\right.
$$


where $\tau_{\alpha}>0$ and $\omega_{\alpha}>0$. The saturation function is as follows:

$$
\operatorname{sat}\left(\alpha_{c}\right)=\left\{\begin{array}{l}
\alpha_{M}, \alpha_{c}>\alpha_{M}, \\
\alpha_{c}, \alpha_{m} \leq \alpha_{c} \leq \alpha_{M}, \\
\alpha_{m}, \alpha_{c}<\alpha_{M},
\end{array}\right.
$$

where $\alpha_{M}>0$ and $\alpha_{m}<0$ are the upper and lower bounds of AOA command, respectively.

Remark 6. In equation (32), the initial values of $\chi_{\alpha 1}$ and $\chi_{\alpha 2}$ are set as $\chi_{\alpha 1}(0)=\alpha(0)$ and $\chi_{\alpha 2}(0)=-\left(\omega_{\alpha}+1\right) \chi_{\alpha 1}(0)$.

The purpose is to make the initial error of AOA zero and meet specific constraint. Compared with Refs. [24-29], there is no need to assume that the initial error satisfies the constraints. And it can be ensured that the AOA command $\alpha_{d}$ meets the limiting condition by assigning an initial value; the relevant proof will be given below.

In order to offset the influence caused by limiting AOA command, the auxiliary compensation system is designed as

$$
\dot{\xi}_{\gamma}=-k_{\gamma \xi} \xi_{\gamma}+g_{\gamma}\left(\operatorname{sat}\left(\alpha_{c}\right)-\alpha_{c}\right),
$$

where $k_{\gamma \xi}>0$.

Combining equations (31) and (34), the virtual control law is designed as

$$
\alpha_{c}=\frac{1}{g_{\gamma}}\left(-k_{\gamma} v_{\gamma}-\frac{g_{\gamma}^{2} v_{\gamma}}{2}-f_{\gamma}-\widehat{d}_{2}+\chi_{\gamma 2}-k_{\gamma \xi} \xi\right),
$$

where $k_{\gamma}>0$ and $\widehat{d}_{2}$ is the LESO estimation of $d_{2}$.

Substituting equations (34) and (35) into equation (31), we get

$\dot{v}_{\gamma}=-k_{\gamma} v_{\gamma}+g_{\gamma} e_{\alpha}+g_{\gamma}\left(\alpha_{d}-\operatorname{sat}\left(\alpha_{c}\right)\right)-\frac{1}{2} g_{\gamma}^{2} v_{\gamma}+d_{2}-\widehat{d}_{2}+\chi_{\gamma 2}-\dot{\gamma}_{d}$.

Step 2. Define the performance function for AOA error

$$
\omega_{\alpha}(t)=\left(\omega_{0}-\varpi_{\infty}\right) \exp (-\mu t)+\varpi_{\infty}
$$

where $\omega_{0}>0, \omega_{\infty}>0, \mu>0$,and $\omega_{0}>\omega_{\infty}$.

According to equation (17), the AOA error is transformed as

$$
\varepsilon_{\alpha}(t)=\frac{1}{2} \ln \left(\frac{1+e_{\alpha} / \varpi_{\alpha}}{1-e_{\alpha} / \varpi_{\alpha}}\right)
$$

Remark 7. This paper constrains the AOA error within the prescribed range by using the prescribed performance method, which can further improve the transient performance of the tracking error while ensuring its steady-state performance.
Combining equations (28) and (8), the derivative of $\varepsilon_{\alpha}$ is given by

$$
\dot{\varepsilon}_{\alpha}=r_{\alpha}\left(f_{\alpha}+q_{d}+e_{q}-d_{2}-\dot{\alpha}_{d}\right)+v_{\alpha}
$$

where

$$
\begin{gathered}
r_{\alpha}=\frac{1}{1-\left(e_{\alpha} / \omega_{\alpha}\right)^{2}} \cdot \frac{1}{\omega_{\alpha}}>0, \\
v_{\alpha}=\frac{1}{1-\left(e_{\alpha} / \omega_{\alpha}\right)^{2}} \cdot \frac{\dot{\omega}_{\alpha}}{\omega_{\alpha}^{2}}
\end{gathered}
$$

The virtual control law is designed as

$$
q_{d}=-k_{\alpha} \varepsilon_{\alpha}-\frac{\omega_{\alpha}^{2}}{r_{\alpha}} \varepsilon_{\alpha}-\frac{\sigma_{\alpha} r_{\alpha}}{4} \varepsilon_{\alpha}-f_{\alpha}-\widehat{d}_{2}+\dot{\alpha}_{d}-\frac{v_{\alpha}}{r_{\alpha}}-\xi_{q},
$$

where $k_{\alpha}>0, \sigma_{\alpha}>0$ and $\xi_{q}$ is the auxiliary variable to be designed.

Substituting equation (41) into equation (39),

$$
\dot{\varepsilon}_{\alpha}=-k_{\alpha} r_{\alpha} \varepsilon_{\alpha}-\left(\omega_{\alpha}^{2}+\frac{\sigma_{\alpha} r_{\alpha}^{2}}{4}\right) \varepsilon_{\alpha}+r_{\alpha}\left(e_{q}-\xi_{q}\right)-r_{\alpha}\left(d_{2}-\widehat{d}_{2}\right) \text {. }
$$

Remark 8. The solution of the problem of AOA constraint is restricting AOA command and tracking error separately in this paper. That is to ensure that $\alpha_{m}<\alpha_{d}<\alpha_{M}$ and $\left|e_{\alpha}\right|<$ $\varpi_{\alpha}(0)$, so that $\alpha \in\left(\alpha_{m}-\varpi_{\alpha}(0), \alpha_{M}+\varpi_{\alpha}(0)\right)$.

Step 3. Define compensation error

$$
v_{q}=e_{q}-\xi_{q} .
$$

Combining equations (28) and (8), the derivative of $v_{q}$ is given by

$$
\dot{v}_{q}=f_{q}+g_{q} \delta_{e}+d_{3}-\dot{q}_{d}-\dot{\xi}_{q} .
$$

Considering the problem of the elevator angular deflection $\delta_{e}$ constraints of amplitude and rate, a constrained command filter is constructed to restrain the desired control law $\delta_{e d}$ and obtain the actual control input:

$$
\left\{\begin{array}{l}
\dot{\chi}_{\delta 1}=\chi_{\delta 2} \\
\dot{\chi}_{\delta 2}=2 \tau_{\delta} \omega_{\delta}\left[H_{\Psi}\left(\frac{\omega_{\delta}^{2}}{2 \tau_{\delta} \omega_{\delta}}\left(\delta_{e d}-\chi_{\delta 1}\right)\right)-\chi_{\delta 2}\right] \\
\delta_{e}=H_{\delta}\left(\chi_{\delta 1}\right)
\end{array}\right.
$$

where $\tau_{\delta}>0$ and $\omega_{\delta}>0$; the specific definitions of $H_{\delta}(\bullet)$ and $H_{\Psi}(\bullet)$ are given by equations (10) and (11).

Remark 9. Compared with Ref. [36], the constrained command filter can ensure that the elevator angular deflection 
$\delta_{e}$ meets the constraint conditions of amplitude and rate. The specific proof will be given below.

In order to offset the influence of input saturation, the auxiliary system is designed:

$$
\dot{\xi}_{q}=-k_{q \xi 1} \xi_{q}-k_{q \xi 2}\left|g_{q}\right| \xi_{q}+g_{q}\left(\delta_{e}-\delta_{e d}\right)
$$

where $k_{q \xi 1}>0$ and $k_{q \xi 2}>0$.

Combining equations (44) and (46), the desired control law is designed as

$$
\delta_{e d}=\frac{1}{g_{q}}\left(-k_{q} v_{q}-f_{q}-\widehat{d}_{3}+\chi_{q 2}-r_{\alpha} \varepsilon_{\alpha}-k_{q \xi 1} \xi_{q}-k_{q \xi 2}\left|g_{q}\right| \xi_{q}\right),
$$

where $k_{q}>0 ; \widehat{d}_{3}$ is the LESO estimation of $d_{3}$; and $\chi_{q 2}$ is the estimation of the derivative of the virtual control law $\dot{q}_{d}$, which can be obtained by the following command filter.

$$
\left\{\begin{array}{l}
\dot{\chi}_{q 1}=\chi_{q 2}, \\
\dot{\chi}_{q 2}=2 \tau_{q} \omega_{q}\left[\frac{\omega_{q}^{2}}{2 \tau_{q} \omega_{q}}\left(q_{d}-\chi_{q 1}\right)-\chi_{q 2}\right],
\end{array}\right.
$$

where $\tau_{q}>0$ and $\omega_{q}>0$.

Substituting equations (46) and (47) into (44) at the same time, we get

$$
\dot{v}_{q}=-k_{q} v_{q}+d_{3}-\widehat{d}_{3}+\chi_{q 2}-\dot{q}_{d}-r_{\alpha} \varepsilon_{\alpha} .
$$

\section{Stability Analysis}

Theorem 10. For the system (7) and (8), by using equations (24) and (47) to limit the control input, it is guaranteed that fuel equivalent ratio $\Phi$ and elevator angular deflection $\delta_{e}$ meet the restricted conditions, respectively, such that $\Phi \in[$ $\left.\Phi_{\min }, \Phi_{\max }\right], \delta_{e} \in\left[\delta_{\min }, \delta_{\max }\right]$, and $\Psi_{e}=\dot{\delta}_{e} \in\left[\Psi_{\min }, \Psi_{\max }\right]$.

Proof. Since $\Phi=H_{\Phi}\left(\Phi_{d}\right)$ and $\delta_{e}=H_{\delta}\left(\chi_{\delta 1}\right)$, it is easy to get $\Phi \in\left[\Phi_{\min }, \Phi_{\max }\right]$ and $\delta_{e} \in\left[\delta_{\min }, \delta_{\max }\right]$ according to the definition of saturation function $H_{\Phi}(\bullet)$ and $H_{\delta}(\bullet)$.

The term

$$
\dot{\chi}_{\delta 2}=2 \tau_{\delta} \omega_{\delta}\left[H_{\Psi}\left(\frac{\omega_{\delta}^{2}}{2 \tau_{\delta} \omega_{\delta}}\left(\delta_{e d}-\chi_{\delta 1}\right)\right)-\chi_{\delta 2}\right]
$$

in equation (45) is transformed as

$$
\dot{\chi}_{\delta 2}+c_{\delta} \chi_{\delta 2}=c_{\delta} H_{\Psi}\left(\frac{\omega_{\delta}^{2}}{c_{\delta}}\left(\delta_{e d}-\chi_{\delta 1}\right)\right)
$$

where $c_{\delta}=2 \tau_{\delta} \omega_{\delta}$. Due to the saturation function $H_{\Psi}(\bullet) \in[$ $\left.\Psi_{\min }, \Psi_{\max }\right]$, equation (51) is satisfied with

$$
c_{\delta} \Psi_{\min } \leq \dot{\chi}_{\delta 2}+c_{\delta} \chi_{\delta 2} \leq c_{\delta} \Psi_{\max }
$$

Multiplying inequality (52) by $\exp \left(c_{\delta} t\right)$, we have

$$
c_{\delta} \Psi_{\min } \exp \left(c_{\delta} t\right) \leq\left(\chi_{\delta 2} \exp \left(c_{\delta} t\right)\right)^{\prime} \leq c_{\delta} \Psi_{\max } \exp \left(c_{\delta} t\right)
$$

Integrating inequality (53) yields

$$
\left\{\begin{array}{l}
\chi_{\delta 2} \geq \Psi_{\min }\left(1-\exp \left(-c_{\delta} t\right)\right)+\chi_{\delta 2}(0) \\
\chi_{\delta 2} \leq \Psi_{\max }\left(1-\exp \left(-c_{\delta} t\right)\right)+\chi_{\delta 2}(0) .
\end{array}\right.
$$

In practice, the upper and lower bounds of $\Psi_{e}$ satisfy $\Psi_{\min }<0$ and $\Psi_{\max }>0$. Taking the initial value $\chi_{\delta 2}(0)=0$, equation (54) can be simplified to

$$
\Psi_{\min } \leq \chi_{\delta 2} \leq \Psi_{\max }
$$

When $\chi_{\delta 1} \in\left(\delta_{\min }, \delta_{\max }\right)$, then $\delta_{e}=H_{\delta}\left(\chi_{\delta 1}\right)=\chi_{\delta 1}$, and $\Psi_{e}=\dot{\delta}_{e} \in\left[\Psi_{\min }, \Psi_{\max }\right]$; when $\chi_{\delta 1} \notin\left[\delta_{\min }, \delta_{\max }\right]$, then $\delta_{e}(t)$ $=\delta_{\min }$ or $\delta_{e}(t)=\delta_{\max }$, such that $\dot{\delta}_{e}=0 \in\left[\Psi_{\min }, \Psi_{\max }\right]$, so $\Psi_{e}=\dot{\delta}_{e} \in\left[\Psi_{\min }, \Psi_{\max }\right]$

Remark 11. The constrained command filter in Ref. [36] constructed for limiting the amplitude and rate of control input is designed as

$$
\left\{\begin{array}{l}
\dot{\chi}_{\delta 1}=\chi_{\delta 2} \\
\dot{\chi}_{\delta 2}=2 \tau_{\delta} \omega_{\delta}\left[H_{\Psi}\left(\frac{\omega_{\delta}^{2}}{2 \tau_{\delta} \omega_{\delta}}\left(H_{\delta}\left(\delta_{e d}\right)-\chi_{\delta 1}\right)\right)-\chi_{\delta 2}\right] \\
\delta_{e}=\chi_{\delta 1} .
\end{array}\right.
$$

If the function $H_{\Psi}(\bullet)$ reaches the saturation point, equation (56) is transformed as

$$
\left\{\begin{array}{l}
\dot{\chi}_{\delta 1}=\chi_{\delta 2} \\
\dot{\chi}_{\delta 2}=2 \tau_{\delta} \omega_{\delta}\left(\Psi_{m}-\chi_{\delta 2}\right) \\
\delta_{e}=\chi_{\delta 1}
\end{array}\right.
$$

where $\Psi_{m}=\Psi_{\min }$ or $\Psi_{m}=\Psi_{\max }$. Obviously, equation (57) cannot ensure that the amplitude of $\delta_{e}$ meets the restricted conditions. Therefore, the constrained command filter constructed in Ref. [36] cannot guarantee that it achieves effective constraint on $\delta_{e}$.

Theorem 12. For the system (7) and (8), under Assumptions 1-5, consider the constraints of AOA and actuators, and use designed control laws (24) and (47). 
(1) The tracking error of the closed-loop system is ultimately uniformly bounded

(2) AOA satisfies $\alpha \in\left(\alpha_{m}-\varpi_{\alpha}(0), \alpha_{M}+\omega_{\alpha}(0)\right)$

Proof. Define Lyapunov function for system as

$$
W=\frac{1}{2}\left(v_{V}^{2}+v_{\gamma}^{2}+\varepsilon_{\alpha}^{2}+v_{q}^{2}\right)
$$

Combining equations (26), (36), (42), and (49), the derivative of $W$ is obtained as

$$
\begin{aligned}
\dot{W}= & v_{V}\left(-k_{V} v_{V}+\tilde{d}_{1}\right)+v_{\gamma}\left(-k_{\gamma} v_{\gamma}+g_{\gamma} e_{\alpha}+g_{\gamma} \eta_{\alpha}-\frac{1}{2} g_{\gamma}^{2} v_{\gamma}+\tilde{d}_{2}+\dot{\eta}_{\gamma}\right) \\
& +\varepsilon_{\alpha}\left(-k_{\alpha} r_{\alpha} \varepsilon_{\alpha}-\left(\omega_{\alpha}^{2}+\frac{\sigma_{\alpha} r_{\alpha}^{2}}{4}\right) \varepsilon_{\alpha}+r_{\alpha} v_{q}-r_{\alpha} \tilde{d}_{2}\right) \\
& +v_{q}\left(-k_{q} v_{q}+\tilde{d}_{3}+\dot{\eta}_{q}-r_{\alpha} \varepsilon_{\alpha}\right),
\end{aligned}
$$

where $\eta_{\gamma}=\chi_{\gamma 1}-\gamma_{d}, \eta_{\alpha}=\alpha_{d}-\operatorname{sat}\left(\alpha_{c}\right)$, and $\eta_{q}=\chi_{q 1}-q_{d}$ are the errors of command filter and $\tilde{d}_{i}=d_{i}-\widehat{d}_{i}(i=1,2,3)$ are the estimation errors of LESO. $\square$

According to Assumption 4, there exist unknown constants $N_{i}(i=1,2,3)>0$, such that $\left|\dot{\eta}_{\gamma}\right| \leq N_{1},\left|\eta_{\alpha}\right| \leq N_{2}$, and $\left|\dot{\eta}_{q}\right| \leq N_{3}$. According to Assumption 5, there exist unknown constants $\tilde{D}_{i}(i=1,2,3)>0$, such that $\left|\tilde{d}_{1}\right| \leq \tilde{D}_{1}, \quad\left|\tilde{d}_{2}\right| \leq \tilde{D}_{2}$, and $\left|\tilde{d}_{3}\right| \leq \tilde{D}_{3}$.

The following inequalities in equation (59) hold:

$$
\left\{\begin{array}{l}
v_{V} \tilde{d}_{1} \leq \frac{k_{V}}{2} v_{V}^{2}+\frac{1}{2 k_{V}} \tilde{d}_{1}^{2}, \\
v_{\gamma} g_{\gamma} e_{\alpha} \leq \frac{1}{4} g_{\gamma}^{2} v_{\gamma}^{2}+e_{\alpha}^{2}, v_{\gamma} g_{\gamma} \eta_{\alpha} \leq \frac{1}{4} g_{\gamma}^{2} v_{\gamma}^{2}+\eta_{\alpha}^{2}, \\
v_{\gamma} \tilde{d}_{2} \leq \frac{k_{\gamma}}{4} v_{\gamma}^{2}+\frac{1}{k_{\gamma}} \tilde{d}_{2}^{2}, v_{\gamma} \dot{\eta}_{\gamma} \leq \frac{k_{\gamma}}{4} v_{\gamma}^{2}+\frac{1}{k_{\gamma}} \dot{\eta}_{\gamma}^{2}, \\
-r_{\alpha} \varepsilon_{\alpha} \tilde{d}_{2} \leq \frac{\sigma_{\alpha}}{4} r_{\alpha}^{2} \varepsilon_{\alpha}^{2}+\frac{1}{\sigma_{\alpha}} \tilde{d}_{2}^{2}, \\
v_{q} \tilde{d}_{3} \leq \frac{k_{q}}{4} v_{q}^{2}+\frac{1}{k_{q}} \tilde{d}_{3}^{2}, v_{q} \dot{\eta}_{q} \leq \frac{k_{q}}{4} v_{q}^{2}+\frac{1}{k_{q}} \dot{\eta}_{q}^{2} .
\end{array}\right.
$$

Equation (38) can be transformed as

$$
\tanh \left(\varepsilon_{\alpha}\right)=\frac{e_{\alpha}}{\omega_{\alpha}}
$$

According to the mean value theorem, $\tanh \left(\varepsilon_{\alpha}\right)=$ $1-\left.\tanh ^{2}\left(\varepsilon_{\alpha}\right)\right|_{\varepsilon_{\alpha}=\varepsilon_{\alpha}^{\prime}} \bullet \varepsilon_{\alpha}$, where $\varepsilon_{\alpha}^{\prime}$ is an unknown constant which lies on the closed interval formed by 0 and $\varepsilon_{\alpha}$. Note that $\left(1-\tanh ^{2}\left(\varepsilon_{\alpha}\right)\right) \leq 1$; equation (61) satisfies

$$
\left(\varepsilon_{\alpha} \omega_{\alpha}\right)^{2} \leq e_{\alpha}^{2}
$$

Combining equations (60) and (62), equation (59) can be simplified to

$$
\begin{aligned}
\dot{W} \leq & -\frac{k_{V}}{2} v_{V}^{2}-\frac{k_{\gamma}}{2} v_{\gamma}^{2}-r_{\alpha} k_{\alpha} \varepsilon_{\alpha}^{2}-\frac{k_{q}}{2} v_{q}^{2}+\frac{1}{2 k_{V}} \tilde{d}_{1}^{2} \\
& +\left(\frac{1}{k_{\gamma}}+\frac{1}{\sigma_{\alpha}}\right) \tilde{d}_{2}^{2}+\frac{1}{k_{q}} \tilde{d}_{3}^{2}+\eta_{\alpha}^{2}+\frac{1}{k_{\gamma}} \dot{\eta}_{\gamma}^{2}+\frac{1}{k_{q}} \dot{\eta}_{q}^{2} .
\end{aligned}
$$

Let $\quad \boldsymbol{\Lambda}=\left[v_{V}, v_{\gamma}, \varepsilon_{\alpha}, v_{q}\right]^{\mathrm{T}} \quad$ and $\quad \bar{k}=\min$ $\left[k_{V} / 2, k_{\gamma} / 2, r_{\alpha} k_{\alpha}, k_{q} / 2\right]^{\mathrm{T}}$. According to the previous analysis, there exists an unknown constant $N_{W}>0$, such that

$$
\frac{1}{2 k_{V}} \tilde{d}_{1}^{2}+\left(\frac{1}{k_{\gamma}}+\frac{1}{\sigma_{\alpha}}\right) \tilde{d}_{2}^{2}+\frac{1}{k_{q}} \tilde{d}_{3}^{2}+\eta_{\alpha}^{2}+\frac{1}{k_{\gamma}} \dot{\eta}_{\gamma}^{2}+\frac{1}{k_{q}} \dot{\eta}_{q}^{2} \leq N_{W} .
$$

Furthermore, equation (63) is obtained as

$$
\dot{W} \leq-\bar{k}\|\boldsymbol{\Lambda}\|_{2}^{2}+N_{W}
$$

When $\|\boldsymbol{\Lambda}\| \geq \sqrt{N_{W} / \bar{k}}$, equation (65) is satisfied with

$$
\dot{W} \leq 0
$$

Therefore, it can be stated that $W$ is bounded and according to the definition of $W . v_{V}, v_{\gamma}, \varepsilon_{\alpha}$, and $v_{q}$ are bounded. According to Remark $3, e_{\alpha}$ is bounded and meets the prescribed conditions by the boundedness of $\varepsilon_{\alpha}$.

When the control inputs exit saturation and the ideal AOA command satisfies the constraint conditions, there are $\Phi-\Phi_{d}=0$ and $\operatorname{sat}\left(\alpha_{c}\right)-\alpha_{c}=0$ for the auxiliary systems (25) and (34), then the auxiliary variables $\xi_{V} \longrightarrow 0$ and $\xi_{\gamma}$ $\longrightarrow 0$, such that $v_{V} \longrightarrow e_{V}, v_{\gamma} \longrightarrow e_{\gamma}$. Therefore, $e_{V}$ and $e_{\gamma}$ are bounded obviously.

According to the auxiliary system (46), we choose the Lyapunov function $W_{\xi}=(1 / 2) \xi_{q}^{2}$ and its derivative is obtained as

$$
\begin{aligned}
\dot{W}_{\xi} & =-k_{q \xi 1} \xi_{q}^{2}-k_{q \xi 2}\left|g_{q}\right| \xi_{q}^{2}+g_{q}\left(\delta_{e}-\delta_{e d}\right) \xi_{q} \\
& \leq-k_{q \xi 1} \xi_{q}^{2}-k_{q \xi 2}\left|g_{q}\right|\left(\xi_{q}^{2}-\frac{\left|\left(\delta_{e}-\delta_{e d}\right) \xi_{q}\right|}{k_{q \xi 2}}\right)
\end{aligned}
$$

When the control input exits saturation, $\delta_{e d} \in\left[\delta_{\min }\right.$, $\left.\delta_{\max }\right]$, notice that $\lim _{\omega_{\delta} \longrightarrow \infty} \chi_{\delta 1}=\delta_{e d}$, and $\delta_{e}=H_{\delta}\left(\chi_{\delta 1}\right)$, so in equation (67), there is $\left(\delta_{e}-\delta_{e d}\right) \in l_{\infty}$. When the auxiliary variable satisfies $\left|\xi_{q}\right| \geq\left|\left(\delta_{e}-\delta_{e d}\right) / k_{q \xi 2}\right|$, equation (67) can 

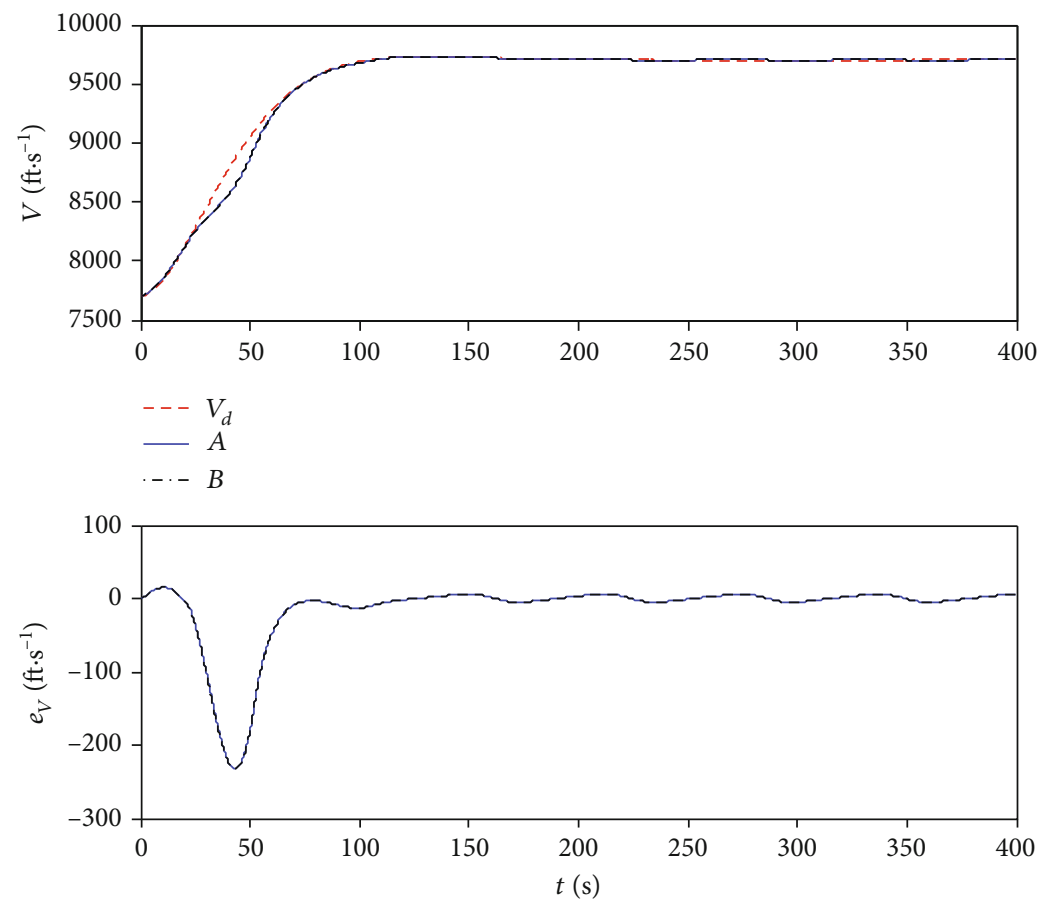

$-A$

Figure 2: Curves of velocity and its tracking error.

be simplified to $\dot{W}_{\xi} \leq-k_{q \xi 1} \xi_{q}^{2}$ obviously. Therefore, $\xi_{q}$ is bounded and $e_{q}$ is bounded.

Equation (32) is transformed as

$$
\ddot{\chi}_{\alpha 1}+2 \tau_{\alpha} \omega_{\alpha} \dot{\chi}_{\alpha 1}+\omega_{\alpha}^{2} \chi_{\alpha 1}=\omega_{\alpha}^{2} \operatorname{sat}\left(\alpha_{c}\right)
$$

Since $\alpha_{m} \leq \operatorname{sat}\left(\alpha_{c}\right) \leq \alpha_{M}$, equation (68) is satisfied with

$$
\alpha_{m} \omega_{\alpha}^{2} \leq \ddot{\chi}_{\alpha 1}+2 \tau_{\alpha} \omega_{\alpha} \dot{\chi}_{\alpha 1}+\omega_{\alpha}^{2} \chi_{\alpha 1} \leq \alpha_{M} \omega_{\alpha}^{2} .
$$

Let $\tau_{\alpha}=1$; multiplying inequality (69) by $\exp \left(\omega_{\alpha} t\right)$, we get

$$
\alpha_{m} \omega_{\alpha}^{2} \exp \left(\omega_{\alpha} t\right) \leq\left(\chi_{\alpha 1} \exp \left(\omega_{\alpha} t\right)\right)^{\prime \prime} \leq \alpha_{M} \omega_{\alpha}^{2} \exp \left(\omega_{\alpha} t\right)
$$

Integrating inequality (70), we get

$$
\left\{\begin{array}{l}
\chi_{\alpha 1} \geq \alpha_{m}\left(1-\mathrm{e}^{-\omega_{\alpha} t}\left(1+\omega_{\alpha} t\right)\right)+\left(1+\omega_{\alpha}\right) \chi_{\alpha 1}+\dot{\chi}_{\alpha 1}(0), \\
\chi_{\alpha 1} \leq \alpha_{M}\left(1-\mathrm{e}^{-\omega_{\alpha} t}\left(1+\omega_{\alpha} t\right)\right)+\left(1+\omega_{\alpha}\right) \chi_{\alpha 1}+\dot{\chi}_{\alpha 1}(0) .
\end{array}\right.
$$

According to the initial assignment of Remark 6, $(1+$ $\left.\omega_{\alpha}\right) \chi_{\alpha 1}+\dot{\chi}_{\alpha 1}(0)=0$, since $\alpha_{M}>0, \alpha_{m}<0$, and $\alpha_{d}=\chi_{\alpha 1}$, equation (71) can be simplified to

$$
\alpha_{m} \leq \alpha_{d} \leq \alpha_{M}
$$

In addition, according to Theorem 10, it can be obtained $\left|e_{\alpha}\right|<\omega_{\alpha}(t)$ by the boundedness of $\varepsilon_{\alpha}$, and furthermore,

$$
-\omega_{\alpha}(0)<e_{\alpha}<\omega_{\alpha}(0)
$$
have

According to equations (72) and (73) and $\alpha=e_{\alpha}+\alpha_{d}$, we

$$
\alpha \in\left(\alpha_{m}-\omega_{\alpha}(0), \alpha_{M}+\omega_{\alpha}(0)\right)
$$

\section{Simulation Results}

In order to verify the effectiveness of the control scheme proposed in this paper, the longitudinal motion model (1)-(5) of HFV is taken as the object, and the controller designed in Section 3 is used to perform MATLAB simulation. The relevant parameters of the HFV model are taken from Ref. [2].

The gains of the controller are chosen as $k_{V}=0.4, k_{h}=$ $0.5, k_{\gamma}=0.2, k_{\alpha}=1, k_{q}=2$, and $\sigma_{\alpha}=0.001$. The parameters of the constrained command filters are chosen as $\tau_{\alpha}=1, \tau_{\gamma}$ $=\tau_{q}=0.8, \tau_{\delta}=0.5, \omega_{\gamma}=\omega_{\alpha}=10, \omega_{q}=20$, and $\omega_{\delta}=90$. The parameters of the prescribed performance are chosen as $\mu$ $=0.1, \omega_{0}=0.01$, and $\omega_{\infty}=0.005$. The parameters of the auxiliary system are chosen as $k_{V \xi}=0.1, k_{\gamma \xi}=0.2, k_{q \xi 1}=$ 0.2 , and $k_{q \xi 2}=0.02$. The parameters of LESO are chosen as $\omega_{V 0}=\omega_{\gamma 0}=\omega_{q 0}=5$. The external disturbances in $d_{1}, d_{2}$, and $d_{3}$ are set as $2 \sin (0.1 t), 0.001 \sin (0.1 t)$, and $0.2 \sin ($ 

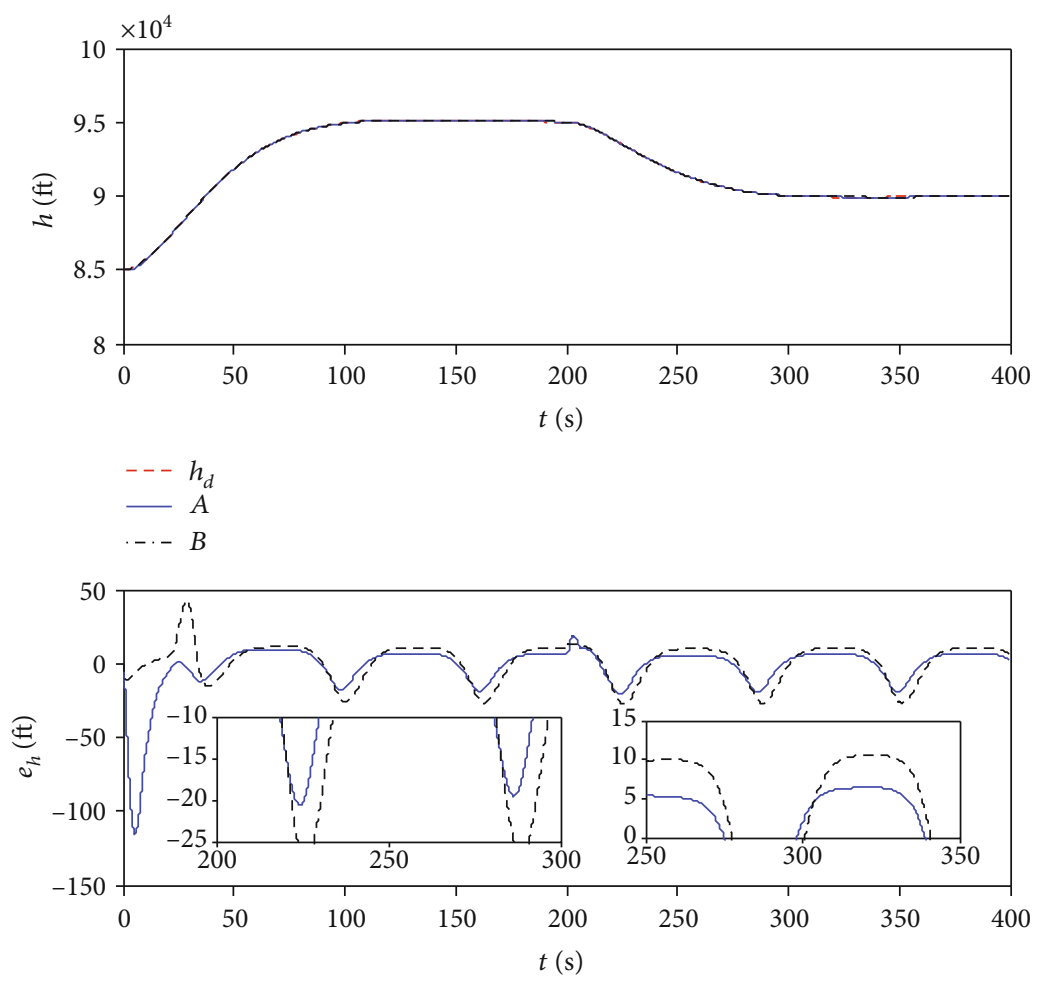

$-A$

$\cdots B$

Figure 3: Curves of height and its tracking error.
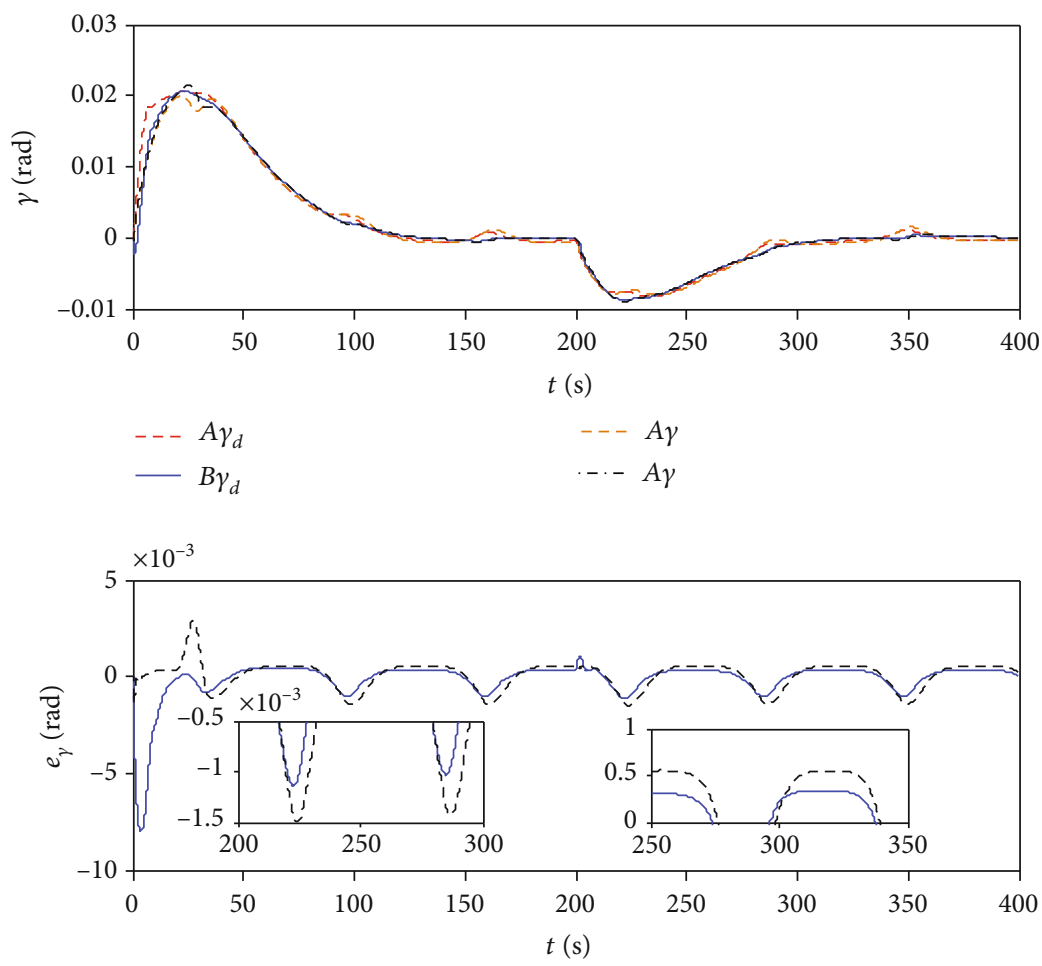

$-A$

FIgURE 4: Curves of fight-path and its tracking error. 

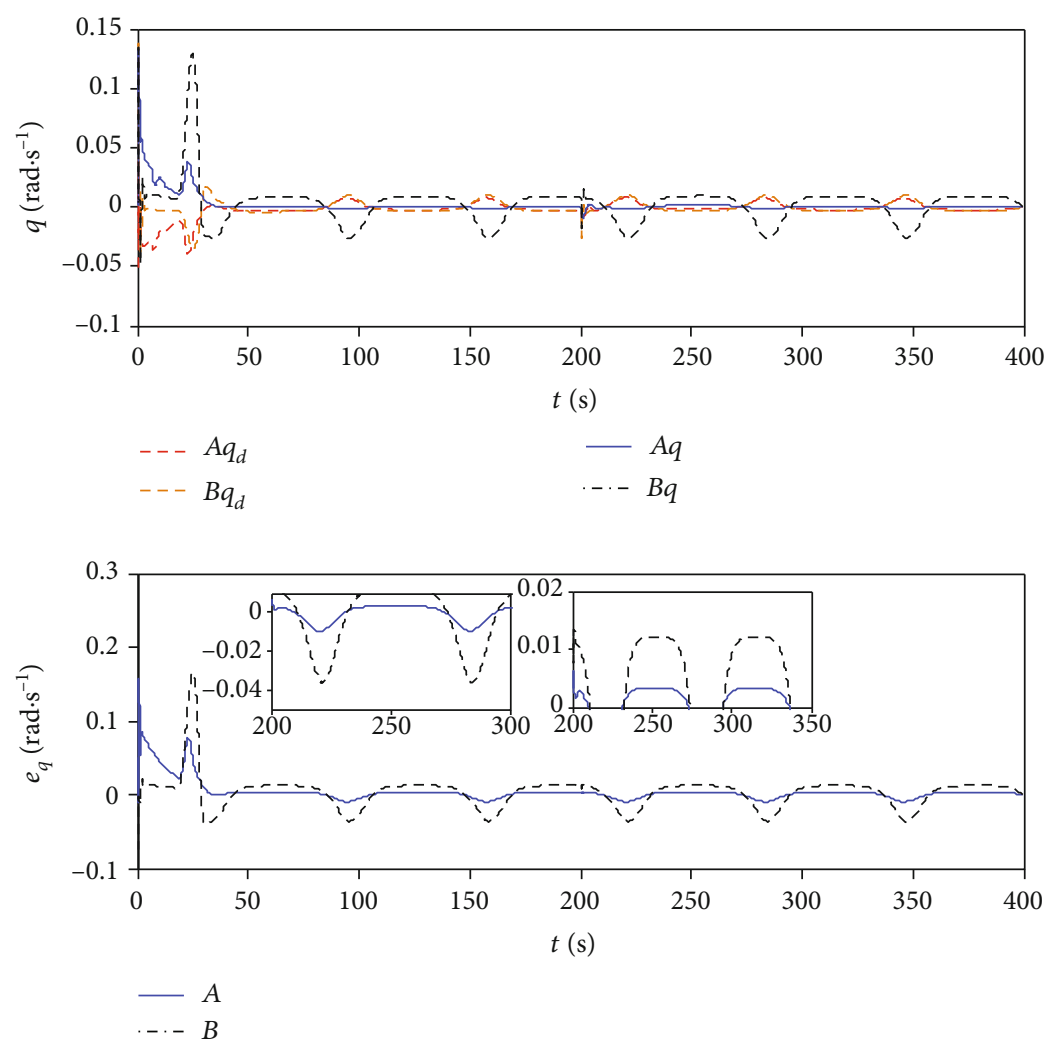

FIgURE 5: Curves of pitch rate and its tracking error.

$0.1 t)$, respectively. We suppose that there is the following perturbation of $30 \%$ in the HFV model

$$
C=C_{0}(1+0.3 \sin (0.1 t))
$$

where $C$ represents the simulation value and $C_{0}$ represents the nominal value.

The initial value of the system states is set as $V_{0}=7702$ $\mathrm{ft} / \mathrm{s}, h_{0}=85000 \mathrm{ft}, \gamma_{0}=0 \mathrm{deg}, \alpha_{0}=1.5153 \mathrm{deg}$, and $q_{0}=0$ $\mathrm{deg} / \mathrm{s}$. We assume the constraint of AOA is set as $\alpha \in\left[-6^{\circ}\right.$, $6^{\circ}$, and the boundaries of saturation function (33) are set as

$$
\left\{\begin{array}{l}
\alpha_{M}=6 \pi / 180-\omega_{\alpha}(0)=0.0947(\mathrm{rad}), \\
\alpha_{m}=-6 \pi / 180+\omega_{\alpha}(0)=-0.0947(\mathrm{rad}) .
\end{array}\right.
$$

The actuator constraints are set as $\Phi \in[0.05,1.5], \delta_{e} \in[$ $\left.-30^{\circ}, 30^{\circ}\right]$, and $\dot{\delta}_{e}=\Psi_{e} \in\left[-100^{\circ} / \mathrm{s}, 100^{\circ} / \mathrm{s}\right]$. The reference commands $V_{d}, h_{d}$ are generated, respectively, by passing step commands $\triangle V_{d}=2000 \mathrm{ft} / \mathrm{s}$ and

$$
\Delta h_{d}= \begin{cases}10000 \mathrm{ft}, & 0<t \leq 200 \mathrm{~s}, \\ -5000 \mathrm{ft}, & t>200 \mathrm{~s},\end{cases}
$$

through the following filter command signal [43]:

$$
G(s)=\frac{0.04^{2}}{s^{2}+0.064 s+0.04^{2}} .
$$

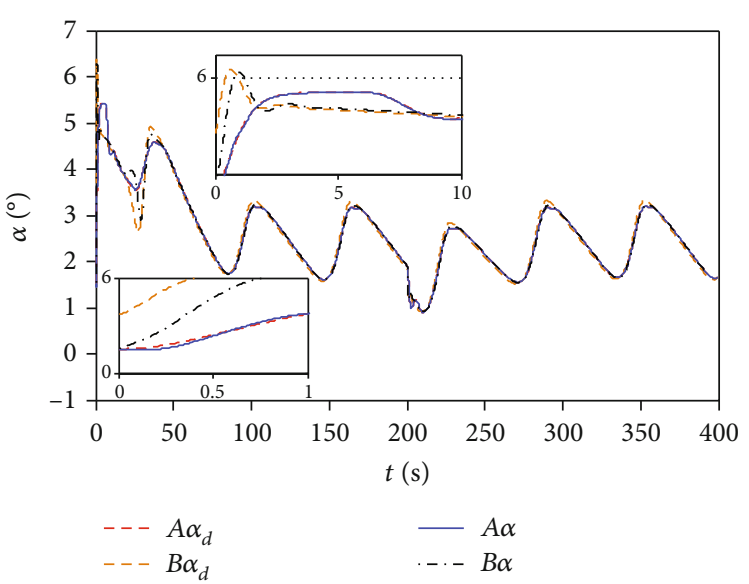

FIgure 6: Tracking curves of AOA.

In order to verify the superiority of the proposed control scheme (denoted as " $\mathrm{A}$ "), the adaptive antisaturation control scheme (denoted as "B") designed in [20] is introduced for comparison and simulation. For the fairness of the comparison, scheme B selects the same control gain parameters as scheme A and adopts LESO with the same parameters to observe the disturbances.

The simulation results are shown in Figures 2-13. It can be seen from Figures 2-7 that both control schemes A and B can guarantee that the system tracks the command signal. It can be seen from Figure 2 that the curves of velocity and its tracking error of scheme A are basically the same as scheme 


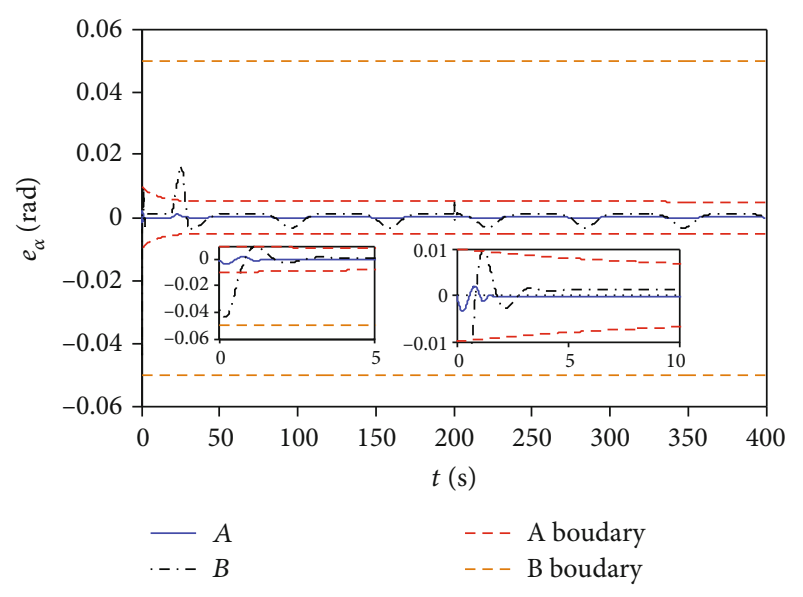

FIgURE 7: Tracking curves of AOA error.

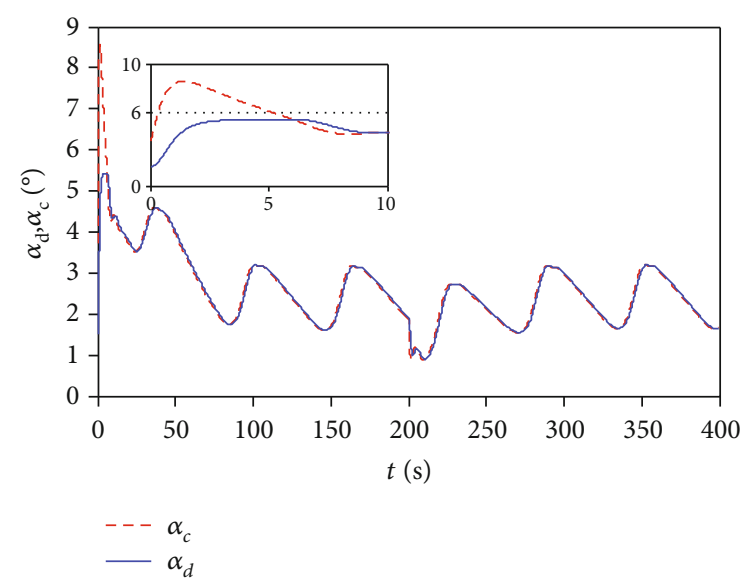

FIGURE 8: Curves of limited AOA command of scheme A.

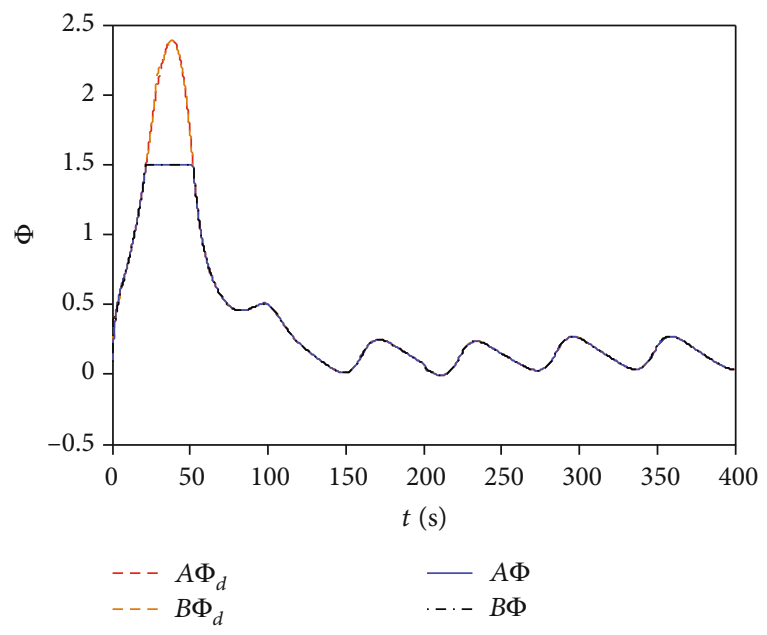

Figure 9: Curves of fuel equivalent ratio.

$\mathrm{B}$, because both schemes $\mathrm{A}$ and $\mathrm{B}$ use the dynamic inverse method in the controller design of velocity subsystem. It can be seen from Figures 3-7 that the tracking accuracy of scheme A is better than scheme B obviously for the altitude

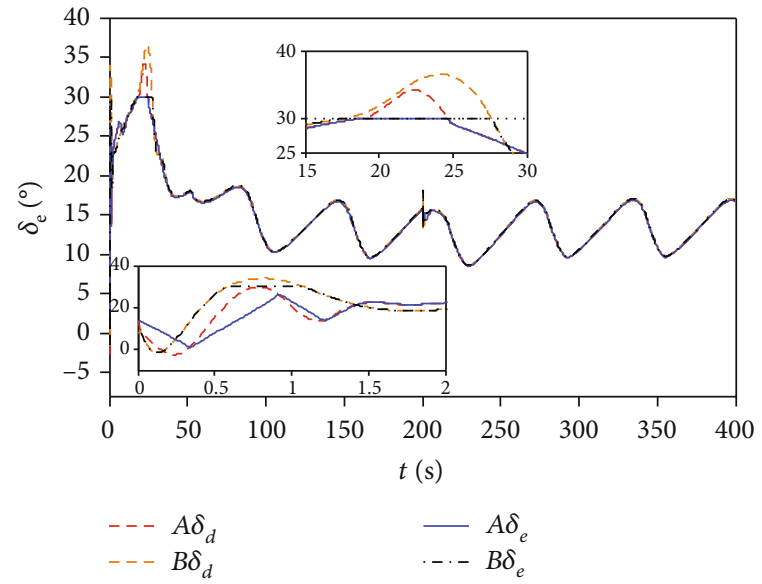

FIgURE 10: Curves of elevator angular deflection.

subsystem. Figure 6 shows that scheme B lacks the limit of the AOA command, which leads to AOA that can achieve command tracking but exceed the constraint range. And it is obvious that AOA of scheme A can track the limiting command stably, and it is always within the constraint range. Figure 7 shows that the constraint range of scheme $\mathrm{B}$ is large which affects the tracking accuracy of AOA, because the range needs to be greater than the initial absolute value of AOA error. And the constraint range is small because the initial value of AOA error is zero in scheme A, which makes AOA error converge to zero quickly. It is the initial value of AOA error that affects the setting of error constraint range, which influences the tracking command effect of AOA and even the whole altitude subsystem (see Figures 3-7).

Remark 13. The solution of the AOA constraint problem by scheme A is mainly due to the following two points. Firstly, there is no need to consider the influence of the initial error on the constraint conditions. So the tracking error converges quickly to zero and meets the prescribed transient and steady-state performance (see Figure 7). Secondly, the AOA command of scheme A is obtained by the constrained command filter, and its amplitude is within the constraint range (see Figure 8). The effectiveness of the constrained command filter is verified.

It can be seen from Figures 9-11 that the amplitude and rate of the control input meet the constraint requirements in scheme A. Although scheme B restricts the amplitude of the control input and the actuator meets the requirements, the rate limitation of the actuator is ignored, and the rate of elevator angular deflection cannot meet the constraint conditions. Figure 12 shows that when the control input is saturated or the desired AOA command exceeds the constraint range, the auxiliary variable responds quickly to compensate the tracking error, which ensures the stability of the system. And the auxiliary variable quickly converges to zero when the system exits saturation. Figure 13 shows that LESO can observe system disturbances effectively, and its estimate is bounded. 


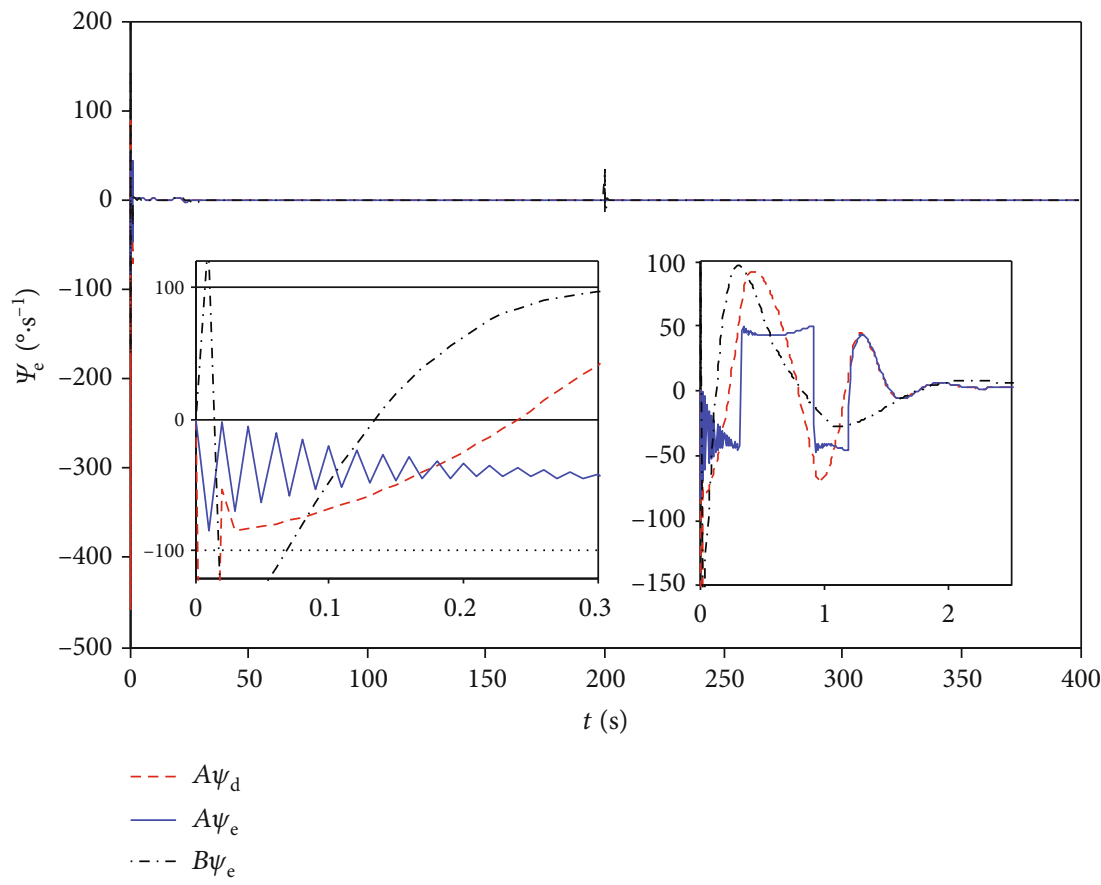

Figure 11: Curves of rate of elevator angular deflection.
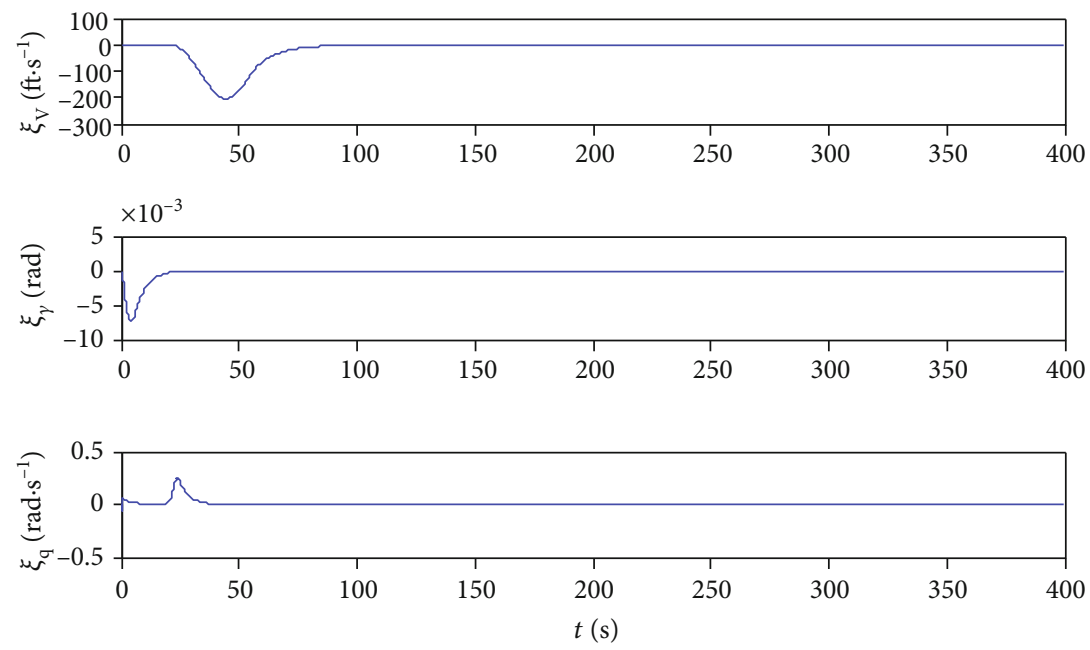

FIgure 12: Curves of auxiliary variable. 

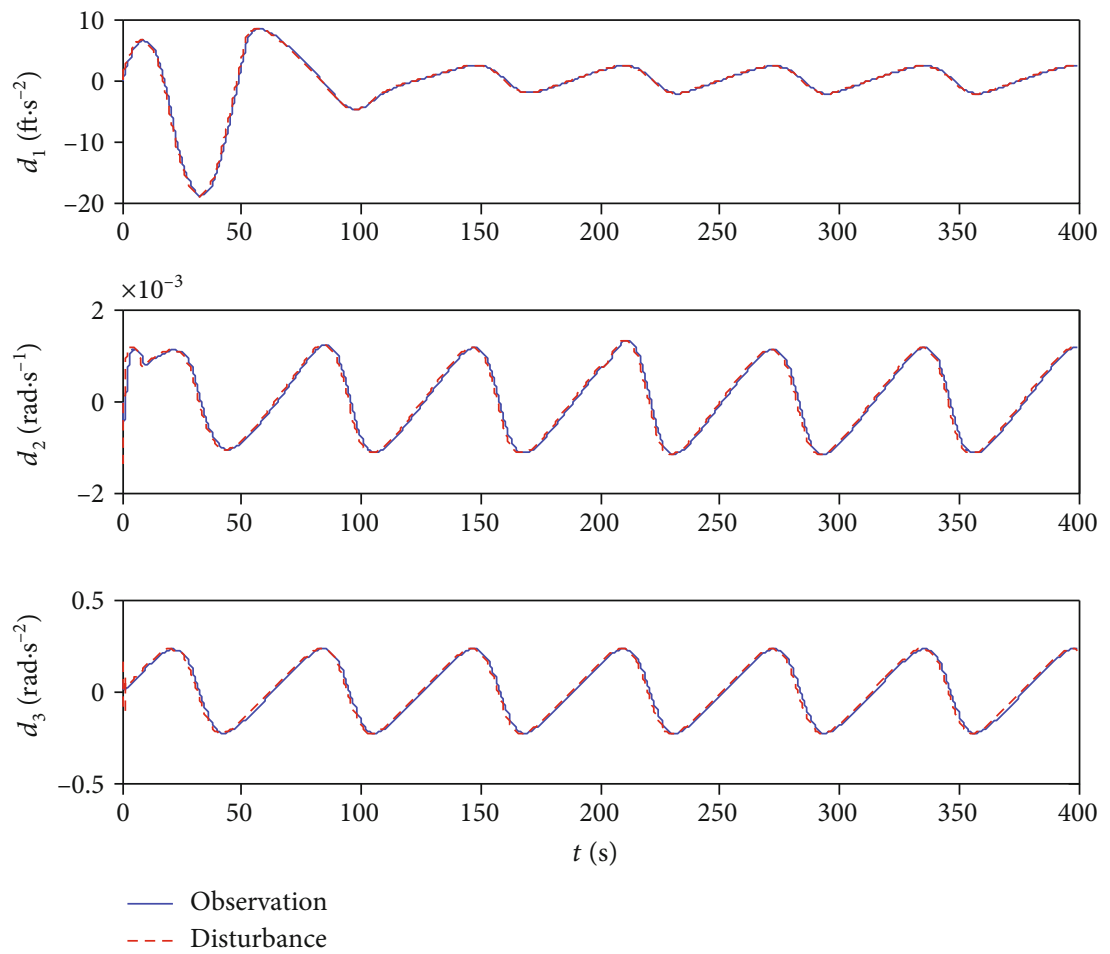

FIgURE 13: Curves of LESO observation.

Remark 14 . We can clearly see that the amplitude and rate of the control input meet the constraint requirements in scheme A. The effectiveness of the constrained command filter limiting the control input is verified. And the problem of actuator constraints is solved effectively.

In summary, the problem of AOA and actuator constraints taking into account parameter perturbation and external disturbance can be solved successfully by the control scheme designed in this paper. And the system achieves a better command tracking effect. The effectiveness of the proposed control scheme is verified by this simulation result.

\section{Conclusions}

An antisaturation backstepping control scheme based on the constrained command filter is proposed for HFV. The AOA constraint problem is solved by combining the proposed constraint command filter and prescribed performance method. AOA meets the constraint during the process of tracking command by constraining the AOA command and tracking error separately. Then, the amplitude and rate of the actuators meet the restricted requirements due to the constrained command filter constructed for the limited input. The problem of AOA and actuators constraint can be solved effectively by the proposed scheme based on the constrained command filter in this paper through theoretical proof and simulation test. In the future research, the methods for controller design of HFV under states constraint and actuator faults will be investigated. Meanwhile, intelligence method will be added in the proposed control scheme.

\section{Data Availability}

The data used to support the findings of this study are available from the corresponding author upon request.

\section{Conflicts of Interest}

The authors declare that there is no conflict of interest regarding the publication of this paper.

\section{Acknowledgments}

This work was supported by the Aeronautical Science Foundation of China (Grant no. 20140184001) and China Postdoctoral Science Foundation (Grant no. 2015M572693).

\section{References}

[1] X. W. Bu and Q. Qi, "Fuzzy optimal tracking control of hypersonic flight vehicles via single-network adaptive critic design," IEEE Transactions on Fuzzy Systems, 2020.

[2] J. T. Parker, A. Serrani, S. Yurkovich, M. A. Bolender, and D. B. Doman, "Control-oriented modeling of an air-breathing hypersonic vehicle," Journal of Guidance, Control, and Dynamics, vol. 30, no. 3, pp. 856-869, 2007.

[3] Y. X. Wang, T. Chao, S. Y. Wang, and M. Yang, "Byrnes-Isidori-based dynamic sliding-mode control for nonminimum phase hypersonic vehicles," Aerospace Science and Technology, vol. 95, p. 105478, 2019.

[4] B. Xu, X. Wang, and Z. K. Shi, "Robust adaptive neural control of nonminimum phase hypersonic vehicle model," IEEE 
Transactions on Systems, Man, and Cybernetics, vol. 51, no. 2, pp. 1107-1115, 2021.

[5] B. H. Lian, H. Bang, and J. E. Hurtado, "Adaptive backstepping control based autopilot design for reentry vehicle," in AIAA Guidance, Navigation, and Control Conference and Exhibit, pp. 1210-1223, Rhode Island, 2004.

[6] X. Y. Zhang, Q. Zong, L. Q. Dou, B. L. Tian, and W. J. Liu, "Improved finite-time command filtered backstepping fault-tolerant control for flexible hypersonic vehicle," Journal of the Franklin Institute, vol. 357, no. 13, pp. 85438565, 2020.

[7] D. Zhao, B. Jiang, and H. Yang, "Backstepping-based decentralized fault-tolerant control of hypersonic vehicles in PDEODE form," IEEE Transactions on Automatic Control, 2021.

[8] Z. H. Yang, W. T. He, Y. S. He, Y. E. Xie, J. Li, and S. Song, "Prescribed performance tracking control for the hypersonic vehicle with actuator faults," International Journal of Aerospace Engineering, vol. 2021, Article ID 8880387, 12 pages, 2021.

[9] P. Li, P. Huang, C. Y. He, and X. Q. Liu, "Finite-time dynamic surface fault-tolerant control for hypersonic vehicle with mismatched disturbances," International Journal of Control, Automation and Systems, vol. 19, no. 7, pp. 2309-2322, 2021.

[10] Y. H. Yang, X. L. Shao, Y. Shi, and W. D. Zhang, "Back-stepping robust control for flexible air-breathing hypersonic vehicle via $\alpha$-filter-based uncertainty and disturbance estimator," International Journal of Control, Automation and Systems, vol. 19, no. 2, pp. 753-766, 2021.

[11] S. Liang, B. Xu, and J. R. Ren, "Kalman-filter-based robust control for hypersonic flight vehicle with measurement noises," Aerospace Science and Technology, vol. 112, article 106566, 2021.

[12] J. L. Sun, J. Q. Yi, Z. Q. Pu, and X. M. Tan, "Fixed-time sliding mode disturbance observer-based nonsmooth backstepping control for hypersonic vehicles," IEEE Transactions on Systems, Man, and Cybernetics, vol. 50, no. 11, pp. 4377-4386, 2020.

[13] J. L. Sun, Z. Q. Pu, J. Q. Yi, and Z. Liu, "Fixed-time control with uncertainty and measurement noise suppression for hypersonic vehicles via augmented sliding mode observers," IEEE Transactions on Industrial Informatics, vol. 16, no. 2, pp. 1192-1203, 2020.

[14] X. W. Bu, Y. Xiao, and H. M. Lei, "An adaptive critic designbased fuzzy neural controller for hypersonic vehicles: predefined behavioral nonaffine control," IEEE/ASME Transactions on Mechatronics, vol. 24, no. 4, pp. 1871-1881, 2019.

[15] R. S. Xia, M. Chen, Q. X. Wu, and Y. H. Wang, "Neural network based integral sliding mode optimal flight control of near space hypersonic vehicle," Neurocomputing, vol. 379, pp. 41$52,2020$.

[16] Y. J. Li, M. S. Hou, S. Liang, and G. Jiao, "Predefined-time adaptive fault-tolerant control of hypersonic flight vehicles without overparameterization," Aerospace Science and Technology, vol. 104, p. 105987, 2020.

[17] X. L. Shao, Y. Shi, and W. D. Zhang, "Input-and-measurement event-triggered control for flexible air-breathing hypersonic vehicles with asymmetric partial-state constraints," Nonlinear Dynamics, vol. 102, no. 1, pp. 163-183, 2020.

[18] Y. Liu, G. Li, Y. C. Li, and Y. H. Wu, "Novel prescribed performance control scheme for flexible hypersonic flight vehicles with nonaffine dynamics and neural approximation," Interna- tional Journal of Aerospace Engineering, vol. 2021, Article ID 8859681, 14 pages, 2021.

[19] R. W. Zuo, Y. H. Li, M. L. Lv, Z. Liu, and Z. C. Liu, "Realization of trajectory precise tracking for hypersonic flight vehicles with prescribed performances," Aerospace Science and Technology, vol. 111, article 106554, 2021.

[20] C. P. Bchlioulis and G. A. Rovithakis, "Prescribed performance adaptive control of SISO feedback linearizable systems with disturbances," in The 16th Mediterranean Conference on Control and Automation, pp. 1035-1040, Ajaccio, France, June 2008.

[21] X. L. Jiao, J. T. Chang, Z. Q. Wang, and D. Yu, "Mechanism study on local unstart of hypersonic inlet at high Mach number," AIAA Journal, vol. 53, no. 10, pp. 3102-3112, 2015.

[22] L. Fiorentini, A. Serrani, M. A. Bolender, and D. B. Doman, "Nonlinear robust adaptive control of flexible air-breathing hypersonic vehicles," Journal of Guidance, Control, and Dynamics, vol. 32, no. 2, pp. 401-416, 2009.

[23] L. Fiorentini and A. Serrani, "Adaptive restricted trajectory tracking for a non-minimum phase hypersonic vehicle model," Automatica, vol. 48, no. 7, pp. 1248-1261, 2012.

[24] H. Y. Qiao, H. Meng, M. J. Wang, W. Ke, and J. G. Sun, “Adaptive control for hypersonic vehicle with input saturation and state constraints," Aerospace Science and Technology, vol. 84, pp. 107-119, 2019.

[25] B. Xu, Z. K. Shi, F. C. Sun, and W. He, "Barrier Lyapunov function based learning control of hypersonic flight vehicle with AOA constraint and actuator faults," IEEE Transactions on Cybernetics, vol. 49, no. 3, pp. 1047-1057, 2019.

[26] J. G. Sun, C. M. Li, Y. Guo, C. Q. Wang, and P. Li, “Adaptive fault tolerant control for hypersonic vehicle with input saturation and state constraints," Acta Astronautica, vol. 167, pp. 302-313, 2020.

[27] Y. Y. Guo, B. Xu, W. X. Han et al., "Robust adaptive control of hypersonic flight vehicle with asymmetric AOA constraint," SCIENCE CHINA Information Sciences, vol. 63, no. 11, pp. 1-13, 2020.

[28] X. N. Tang, D. Zhai, and X. J. Li, “Adaptive fault-tolerance control based finite-time backstepping for hypersonic flight vehicle with full state constrains," Information Sciences, vol. 507, pp. 53-66, 2020.

[29] Y. LIU, C. DONG, W. ZHANG, and Q. WANG, "Phase plane design based fast altitude tracking control for hypersonic flight vehicle with angle of attack constraint," Chinese Journal of Aeronautics, vol. 34, no. 2, pp. 490-503, 2021.

[30] C. X. Luo, H. M. Lei, D. Y. Zhang, and X. J. Zou, “Adaptive neural control of hypersonic vehicles with actuator constraints," International Journal of Aerospace Engineering, vol. 2018, 15 pages, 2018.

[31] J. G. Sun and S. M. Song, "Tracking control of hypersonic vehicles with input saturation based on fast terminal sliding mode," International Journal of Aeronautical and Space Sciences, vol. 20, no. 2, pp. 493-505, 2019.

[32] H. L. Chen, J. Zhou, M. Zhou, and B. Zhao, "Nussbaum gain adaptive control scheme for moving mass reentry hypersonic vehicle with actuator saturation," Aerospace Science and Technology, vol. 91, pp. 357-371, 2019.

[33] C. Y. Dong, Y. Liu, and Q. Wang, "Barrier Lyapunov function based adaptive finite-time control for hypersonic flight vehicles with state constraints," ISA Transactions, vol. 96, pp. $163-176,2020$. 
[34] Y. B. Ding, X. G. Wang, Y. L. Bai, and N. G. Cui, "Novel antisaturation robust controller for flexible air-breathing hypersonic vehicle with actuator constraints," ISA Transactions, vol. 99, pp. 95-109, 2020.

[35] X. L. Shao, Y. Shi, and W. D. Zhang, "Fault-tolerant quantized control for flexible air-breathing hypersonic vehicles with appointed-time tracking performances," IEEE Transactions on Aerospace and Electronic Systems, vol. 57, no. 2, pp. 3003$3015,2021$.

[36] S. Zhang, Q. Wang, and C. Y. Dong, "A novel adaptive dynamic surface control scheme of hypersonic flight vehicles with thrust and actuator constraints," Transactions of the Institute of Measurement and Control, vol. 40, no. 4, pp. 13621374, 2018.

[37] C. X. Luo, H. M. Lei, J. Li, and C. J. Zhou, "A new adaptive neural control scheme for hypersonic vehicle with actuators multiple constraints," Nonlinear Dynamics, vol. 100, no. 4, pp. 3529-3553, 2020.

[38] Y. Li, C. L. Wang, and Q. L. Hu, “Adaptive control with prescribed tracking performance for hypersonic flight vehicles in the presence of unknown elevator faults," International Journal of Control, vol. 92, no. 7, pp. 1682-1691, 2019.

[39] X. Wang, J. Guo, S. J. Tang, and S. Qi, "Fixed-time disturbance observer based fixed-time back-stepping control for an airbreathing hypersonic vehicle," ISA Transactions, vol. 88, pp. 233-245, 2019.

[40] J. A. Farrell, M. Polycarpou, M. Sharma, and Wenjie Dong, "Command filtered backstepping," IEEE Transactions on Automatic Control, vol. 54, no. 6, pp. 1391-1395, 2009.

[41] Z. Q. GAO, "Active disturbance rejection control: a paradigm shift in feedback control system design," in the 2006 American Control Conference, Minneapolis, MN, USA, June, 2006.

[42] X. L. Shao and H. L. Wang, "Performance analysis on linear extended state observer and its extension case with higher extended order," Control and Decision, vol. 30, no. 5, pp. 815-822, 2015.

[43] Z. H. Wu, J. C. Lu, J. P. Shi, Q. Zhou, and X. B. Qu, “Tracking error constrained robust adaptive neural prescribed performance control for flexible hypersonic flight vehicle," International Journal of Advanced Robotic Systems, vol. 14, no. 1, pp. 1-16, 2017. 